\title{
Emotional faces and the default mode network
}

Sreenivas, S.; Boehm, S.G.; Linden, D.E.

\section{Neuroscience Letters}

\author{
DOI: \\ 10.1016/j.neulet.2011.11.012
}

Published: 11/01/2012

Peer reviewed version

Cyswllt i'r cyhoeddiad / Link to publication

Dyfyniad o'r fersiwn a gyhoeddwyd / Citation for published version (APA):

Sreenivas, S., Boehm, S. G., \& Linden, D. E. (2012). Emotional faces and the default mode network. Neuroscience Letters, 506(2), 229-234. https://doi.org/10.1016/j.neulet.2011.11.012

\footnotetext{
Hawliau Cyffredinol / General rights

Copyright and moral rights for the publications made accessible in the public portal are retained by the authors and/or other copyright owners and it is a condition of accessing publications that users recognise and abide by the legal requirements associated with these rights.

- Users may download and print one copy of any publication from the public portal for the purpose of private study or research.

- You may not further distribute the material or use it for any profit-making activity or commercial gain

- You may freely distribute the URL identifying the publication in the public portal ?
}

Take down policy

If you believe that this document breaches copyright please contact us providing details, and we will remove access to the work immediately and investigate your claim. 


\section{Accepted Manuscript}

Title: Emotional faces and the default mode network

Authors: S. Sreenivas, S.G. Boehm, D.E.J. Linden

PII:

S0304-3940(11)01534-5

DOI: $\quad$ doi:10.1016/j.neulet.2011.11.012

Reference: $\quad$ NSL 28466

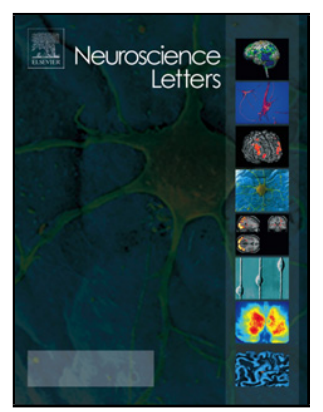

To appear in: $\quad$ Neuroscience Letters

Received date: $\quad 9-7-2011$

Revised date: $\quad$ 13-10-2011

Accepted date: $\quad$ 5-11-2011

Please cite this article as: S. Sreenivas, S.G. Boehm, D.E.J. Linden, Emotional faces and the default mode network, Neuroscience Letters (2010), doi:10.1016/j.neulet.2011.11.012

This is a PDF file of an unedited manuscript that has been accepted for publication. As a service to our customers we are providing this early version of the manuscript. The manuscript will undergo copyediting, typesetting, and review of the resulting proof before it is published in its final form. Please note that during the production process errors may be discovered which could affect the content, and all legal disclaimers that apply to the journal pertain. 
- Areas of the default-mode network were deactivated during processing of emotional faces

- Levels of deactivation differed across emotions, with happy least deactivating

- This deactivation was mirrored by fronto-parietal activation

- Correlation analyses revealed different patterns of connectivity for emotion categories 
Emotional faces and the default mode network Sreenivas, Boehm, \& Linden

\title{
Emotional faces and the default mode network
}

\author{
Authors: Sreenivas, $\mathrm{S}^{1}$, Boehm, S. G ${ }^{2}$ and Linden, D. E. $\mathrm{J}^{3}$ \\ ${ }^{1}$ North Wales Organisation for Randomised Trials in Health (\& Social Care), Bangor \\ University, Bangor, UK \\ ${ }^{2}$ Wolfson Centre for Clinical and Cognitive Neuroscience, School of Psychology, Bangor \\ University, Bangor, UK \\ ${ }^{3}$ Dept. of Psychological Medicine \& Neurology and School of Psychology, Cardiff \\ University, Cardiff, UK
}

Corresponding author: Professor David Linden, Henry Wellcome Building, Cardiff University School of Medicine, Cardiff, CF144XN; Phone: +44 2920687928; Email: lindend@,cardiff.ac.uk

\begin{abstract}
The default-mode network (DMN) of the human brain has become a central topic of cognitive neuroscience research. Although alterations in its resting state activity and in its recruitment during tasks have been reported for several mental and neurodegenerative disorders, its role in emotion processing has received relatively little attention. We investigated brain responses to different categories of emotional faces with functional magnetic resonance imaging (fMRI) and found deactivation in ventromedial prefrontal cortex (VMPFC), posterior cingulate gyrus (PC) and cuneus. This deactivation was modulated by emotional category and was less prominent for happy than for sad faces. These deactivated areas along the midline conformed to areas of the DMN. We also observed emotion-dependent deactivation of the left middle frontal gyrus, which is not a classical component of the DMN. Conversely, several areas in a fronto-parietal network commonly linked with attention were differentially activated by emotion categories. Functional connectivity patterns, as obtained by correlation of activation levels, also varied between emotions. VMPFC, PC or cuneus served as hubs between the DMN-type areas and the fronto-
\end{abstract}


Emotional faces and the default mode network Sreenivas, Boehm, \& Linden

parietal network. These data support recent suggestions that the DMN is not a unitary system but differentiates according to task and even type of stimulus. The emotion-specific differential pattern of DMN deactivation may be explored further in patients with mood disorder, where the quest for biological markers of emotional biases is still ongoing.

Key words: emotion; connectivity; functional imaging study; precuneus; deactivation

\begin{abstract}
Abbreviations: DMN: Default mode network; fMRI: Functional magnetic resonance imaging; BOLD: Blood oxygenation level dependant; SAM: Self assessment manikins; EFT: Emotional face task; M: Group mean; SD: Standard deviation; RT: Reaction time; ROIs: Regions of interest; VMPFC: Ventro medial prefrontal cortex; PC: Posterior cingulate gyrus; LMFG: Left middle frontal gyrus; RIFG: Right inferior frontal gyrus; LIFG; Left inferior frontal gyrus; LIPL: Left inferior parietal lobe
\end{abstract}


Emotional faces and the default mode network Sreenivas, Boehm, \& Linden

\section{Introduction}

The default-mode network (DMN) of the human brain, which is active during the resting state but is deactivated during task performance $[5,10,11,14,15,23,24,29]$ includes cortical midline structures such as VMPFC and PC, and areas in the lateral parietal cortex and superior temporal gyrus bilaterally $[7,11,15,24,30]$. The functional role of this intriguing activation pattern, with deactivation scaled with cognitive load [19], is still under investigation. Specific functions that have been suggested to be supported by the DMN include self-referential thought [3] and mind wandering [12]. Another matter of current investigation is whether the DMN is a unitary system or functionally specialised, both in terms of its activation and deactivation [22]. There are three main ways of localising the DMN, through the deactivations observed during parametric manipulation of cognitive load, through the manipulation of self-relatedness or through data-driven analyses of resting state data [12].

Dysfunction of the DMN has been implicated in several neuropsychiatric disorders [27], including schizophrenia $[8,20,25,26]$ and mood disorders [11], and in cognitive decline associated with dementia [10]. Although such altered connectivity patterns in the DMN in mood disorders [11] suggest a role of this system in emotion regulation [30], its responses to different categories of emotional stimuli have rarely been investigated [11]. The present study therefore assessed brain activity in response to different categories of emotional stimuli in healthy participants with a particular focus on connectivity patterns both within the DMN and between the DMN and other areas. The aim of the study was to contribute to the understanding of brain activity patterns of the processing of the different categorical emotions. Because we did not employ an independent functional localiser of the DMN in this study, we supplemented our whole-brain analysis with a region-of-interest analysis on DMN foci obtained from previous studies.

\section{Method}

\section{Participants}

We recruited 15 healthy participants ( 10 females; 14 right handed) from an academic environment. Their age ranged from 20-37 with a mean age of 28 years. Additional 14 
Emotional faces and the default mode network Sreenivas, Boehm, \& Linden

participants (nine females; 14 right handed) from similar academic environment were recruited who participated only in a behavioural control experiment. They were in the age range of 19-34 with a mean age of 24 years. The study was approved by the ethics committee of the School of Psychology of Bangor University and participants gave written informed consent prior to participation.

\section{Exclusion criteria}

The usual exclusion criteria for research functional magnetic resonance imaging (fMRI) scanning (e.g., claustrophobia, pregnancy, cardiac history, high blood pressure, ferromagnetic implants) applied. None of the participants had a history of psychiatric or neurological disorders.

\section{Materials}

We used 40 [5 emotions-angry, happy, sad, fearful, neutral x 8 individuals (4 male, 4 female)] emotional faces (Ekman P, Friesen WV (1979): Pictures of Facial Affect. Palo Alto, CA: Consulting Psychologists). Participants rated their emotional responses evoked by these faces along the dimensions of valence and arousal with the Self Assessment Manikin (SAM) [2] before viewing them during fMRI scanning.

\section{Procedure}

a) Rating scales:

We investigated participants' induced arousal and valence using a computerised version of the SAM [2]. We showed faces of 8 persons for each of the 5 emotions that were to be rated on a nine point scale for arousal and valence respectively. Stimuli were controlled by the E prime software (Psychology software tools Inc, Sharpsburg, PA, USA).

b) fMRI paradigm:

Participants were scanned during an emotional faces task (EFT). Each participant underwent two runs of the EFT, consisted of 24 blocks of the 6 conditions ( 5 emotions and rest), each occurring 4 times. The order of blocks was randomized. Each emotion block started with a fixation cross for $500 \mathrm{~ms}$, followed by a word denoting the emotion category (1500 ms) and eight faces. Each of the 8 faces appeared for $1500 \mathrm{~ms}$, and a fixation cross was presented again for 500 $\mathrm{ms}$ between face presentations and for $2 \mathrm{~s}$ at the end of the block. These block alternated with $20 \mathrm{~s}$ 
Emotional faces and the default mode network Sreenivas, Boehm, \& Linden

fixation (baseline) blocks. In each emotional block we randomly interspersed occasional nonmatching faces (a sad face in the neutral block and a neutral face within all other blocks), with a non-match appearing on average in every second block. Faces subtended a visual angle of $7^{\circ}$ in width and $11^{\circ}$ in height. Participants responded by pressing the left button for facial expressions that matched the cued emotion and the right button for those that did not. The assignment of hands to response was counterbalanced across participants.

We presented stimuli on a video projector, back-projecting into the scanner room through a mirror, and controlled stimulus presentation from a Dell Latitude D610 laptop with the Presentation software (Neurobehavioral Systems, Inc., Albany, CA). Because of technical difficulties the behavioural responses from the EFT were not obtained from the scanning sessions, but from 14 additional participants outside the scanner.

\section{c) fMRI parameters}

We scanned participants on a Philips Gyroscan Intera 1.5 Tesla system with the following parameters: repetition time, $2000 \mathrm{~ms}$; echo time, $40 \mathrm{~ms}$; flip angle, $90^{\circ}$; matrix size, 64x64; field of view, 192mm x 192mm; pixel size, $3 \mathrm{~mm}$ x 3mm; slice thickness 5mm; 20 slices. The functional time-series for EFT consisted of 260 volumes. We also acquired a high resolution (1mm x 1mm x 1mm) 3-dimensional T1 sequence for anatomical alignment.

\section{Data Analysis}

Rating scales

We computed the descriptive statistics for the behavioural response on the SAM to document the group mean (M) and standard deviation (SD) for Arousal and Valence. Supplementary behavioural data: We compared the number of hits for non-match detection across emotions and reaction times (RTs) for the EFT behavioural data with a 1-way repeated measures ANOVA and t-tests and Bonferroni correction for multiple comparisons. fMRI data

fMRI data was pre-processed with the Brain Voyager QX software (Braininnovation, Maastricht, the Netherlands). The pre-processing procedures to remove the artefacts resulting from factors such as physiological noise included linear trend removal and temporal high pass 
Emotional faces and the default mode network Sreenivas, Boehm, \& Linden

filtering (low cut off: 2 cycles per run). The anatomical and functional data was normalised into the Talairach coordinate system (resampling the functional data into $3 \times 3 \times 3 \mathrm{~mm}^{3}$ voxels, and we spatially smoothed the functional data with a $4 \mathrm{~mm}$ full width at half maximum Gaussian kernel to enable analysis across participants. We computed a General linear model for the experiment with 11 predictors including the five experimental conditions (5 emotion categories) and six motion confounds (translation along the three axes and angular movement through the three angles of a Cartesian coordinate system). The predictors for the experimental conditions were obtained by convolving the duration of each condition (from cue to end of last stimulus) with a $2 \gamma$ haemodynamic response function as implemented in BrainVoyager. We performed a randomeffects analysis with a repeated measures ANOVA. We report areas with a significant maineffect for category (cluster threshold corrected $=297 \mathrm{~mm}^{3} ; \mathrm{p}<.01$, using the cluster threshold plugin of Brainvoyager, which estimates cluster level thresholds for a given $\mathrm{p}$-value with a Monte Carlo simulation). The beta-values for the thus-identified regions of interest (ROIs) were extracted for further statistical analysis with Statistical Package for Social Sciences (SPSS) (SPSS Inc., Chicago, Illinois). We compared the extracted beta values from ROIs for the five emotions using t-tests and Bonferroni correction for multiple comparisons.

In this study we defined the DMN based on task-related deactivations, which is one of the standard procedures $[12,24]$. However, to confirm that the emotion-specific deactivation patterns were actually observed in independently localised canonical DMN regions we defined regions of interest (ROIs) based on published studies that defined the DMN by other means (self-referential thought [6], independent components analysis of resting state data [23]) and computed the same contrasts as above for the mean beta values from these ROIs (Fig.1).

\section{Correlation Analysis}

To analyse the relationship between the ROIs, which showed a significant main-effect for emotion, a correlation analysis (Pearson) was conducted on the beta-values extracted from these ROIs. The ROIs were graphically mapped on the basis of the significant correlation outcome. We used this graphical representation of the resultant significant correlations to identify the emotion 
Emotional faces and the default mode network

Sreenivas, Boehm, \& Linden

specific networks between the ROIs. This networking represents the functional-connectivity between the ROIs in this study.

\section{Results}

SAM:

The rating data (Table 1) confirmed that the facial expression induced the expected emotional states in participants. Positive values denote higher arousal and more positive valence. EFT results

Imaging:

A significant effect for category was found in bilateral ventromedial prefrontal cortex (VMPFC), posterior cingulate gyrus (PC), cuneus, right inferior frontal gyrus (RIFG), left inferior frontal gyrus (LIFG), left middle frontal gyrus (LMFG) and left inferior parietal lobe (LIPL). This main-effect for category was driven mainly by the differences between happy and other conditions (table 2, fig. 1). The follow-up contrast to this main-effect of emotion showed that deactivation for happy faces was smaller compared to anger and/or sad in the VMPFC, PC, Cuneus and LMFG and lower activation in the RIFG, LIFG and LIPL.

Correlation analysis to assess functional connectivity:

The different connectivity patterns for each emotion are documented in Figure 2. The most common network across emotions was the medial-network consisting of VMPFC, PC and cuneus, except for the fear condition, where PC was not found in the medial-network. Instead, the network for the fear condition included the LMFG, another deactivated area (Fig. 1).

For anger, we additionally found a triangular connectivity between LIPL, LIFG and RIFG, which were all activated compared to baseline (Fig. 1).

Neutral faces elicited a more complex network. Again, we observed the medial-network including VMPFC, PC and cuneus. However, cuneus activity was correlated with LMFG, which in turn formed another network with LIPL, LIFG and RIFG.

The functional-network for the happy faces had an even more complex structure. The PC, LMFG and LIPL were three main hubs, which connected between themselves and other subnetworks. The medial-network was preserved with VMPFC, cuneus and PC but also included 
Emotional faces and the default mode network Sreenivas, Boehm, \& Linden

LMFG. Another sub-network connected with the PC included LIFG and LIPL. The LIFG and LIPL were also connected to RIFG forming another triangular sub-network.

Supplementary behavioral data

The repeated measures analysis of variance (1-factorial) revealed no significant difference between the numbers of hits (Table 1) in response to each emotion. However, there was significant difference between the RTs (Table 1) for the different categories, F $(4,52)$ $=19.05, \mathrm{p}=.001$. The follow up contrasts revealed that the RT for happy faces was significantly faster than for all other categories (happy vs neutral, $\mathrm{p}<.001$; sad \& angry, $\mathrm{p}=.001$; fear, $\mathrm{p}<.05$; bonferroni correction for multiple comparisons). The SAM data from the original healthy group was compared to the supplementary behavioural data to assess the similarity of the groups. The analysis of variance revealed no significant difference between the groups.

\section{Cross study ROI comparison}

The coordinates of the references ROIs from the two published DMN studies (VMPFC $(7,54,6)[6]$; cuneus $(-3,-49,22)[23])$ were included in the activated clusters from this study and the cluster centers were in close proximity (Table 2). The significant contrasts [VMPFC (happy-sad, fear); cuneus (sad-happy, angry, fear)] on the beta values from these ROIs replicated those obtained by the whole-brain analysis (Fig. 1).

\section{Discussion}

This study investigated the brain activations and deactivations in response to emotional faces from different categories. We report deactivation of VMPFC, PC, cuneus, LMFG and activation of LIFG, RIFG, and LIPL across emotions, but also important differences between different emotion categories in these areas.

The deactivations closely resembled the DMN reported by previous studies, which prominently included VMPFC, PC and cuneus [3, 10, 15, 17]. However, the middle frontal gyrus is not normally part of the DMN, and its deactivation in the present task may be related to the specific emotional nature of stimuli. The cross study ROI comparison confirmed the ROIs identified in this study to be part of the DMN. The study by Mayberg [6] identified VMPFC 
Emotional faces and the default mode network Sreenivas, Boehm, \& Linden

activity during self-referential thoughts that was in close proximity to the VMPFC coordinates of our study. Similarly the resting state study by Rotarska-Jagiela [23] identified the cuneus with similar coordinates to our study. Importantly, differences between emotion categories for these independently localized DMN areas replicated our results from the whole-brain analysis (Fig.1). No previous studies reported DMN deactivation during emotional categorisation in a healthy group. However, significant negative BOLD responses were found in the perigenual anterior cingulate cortex, VMPFC and dorsal PC in both depressed and healthy individuals during an emotional task in which they had to judge whether the picture was positive or negative [11].

Deactivation of classical DMN areas during emotion perception might seem counterintuitive. Models of emotion processing posit a strong self-referential component [15], and thus the DMN, if it is indeed involved in self-referential thought, should have been activated rather than deactivated. However, patterns of deactivation in fMRI are always computed in comparison with a baseline, which contains uncontrollable mental activity that may very well include self-referential thoughts as well. The differential pattern of deactivation is therefore more interesting. One previous study demonstrated a negative correlation between measures of emotions and induced activity during rest within regions of the DMN, including the VMPFC [30].

In our study the DMN was least deactivated for the happy condition. Because hit rates did not differ across conditions we do not believe that this is merely an effect of a lower cognitive load in that condition. However, this reduced deactivation may indicate a more automatic mode of processing of happy faces in healthy individuals (16). If DMN deactivation is an indicator of effortful task processing [21,30], this might indicate that happy faces are least disruptive of ongoing mental activity and demand the least attention. This interpretation would be supported by the differences in reaction times (responses for mismatch detection were fastest for happy faces). The mirrored pattern of activation (lowest for happy) in fronto-parietal areas that form part of the attention network would also support such an account. However, the present design could not disentangle the causes and consequences of DMN deactivation. 
Emotional faces and the default mode network Sreenivas, Boehm, \& Linden

The second main network identified by the analysis comprised RIFG, LIFG and LIPL. These areas are part of the fronto-parietal attention networks [4, 23] and their activation may be related to the attention demands of the concurrent mismatch detection task. The inferior frontal gyrus is centrally involved in the "bottom-up" attention network for detection of changes in the environment $[1,4,9,18]$.

Although the function of the DMN has so far remained elusive, its coordinated (albeit often anticorrelated) activity with the fronto-parietal "task-positive" network seems to be important for normal brain function and can be disrupted in mental illness and with related symptoms $[8,13,26,27,28]$. Hubs for this coordination are of particular interest, and in our study were located in the VMPFC, PC, or cuneus for different emotion categories.

\section{Conclusion}

This study reports deactivation of the DMN during the processing of emotional faces, and differential deactivation levels and functional-connectivity patterns across emotional categories. We found the least deactivation for happy faces, which we interpret as indicating a more automatic response mode to positive stimuli, and the strongest deactivation for negative categories, which may indicate their higher evolutionary saliency. We propose that emotionspecific connectivity patterns of the DMN may be considered as potential future markers of mood states.

Acknowledgement: This study was supported by the Economic and Social Research Council (ESRC grant - RES-062-23-0946) to DL and a Research Councils UK (RCUK) Academic Fellowship to SB. No conflict of interest reported. 
Emotional faces and the default mode network

\section{References}

[1] Bledowski C, Prvulovic D, Goebel R, Zanella F E, Linden D E J, Attentional systems in target and distractor processing: a combined ERP and fMRI study, Neuroimage. 22 (2004) $530-40$.

[2] Bradley M M, \& Lang P J, Measuring emotion: The Self-Assessment Manikin and the semantic differential, Journal of Behavior Therapy and Experimental Psychiatry. 25 (1994) $49-59$.

[3] Buckner R L, Andrews-Hanna J R, \& Schacter D L, The brain's default network, anatomy, function, and relevance to disease, Ann. N. Y. Acad. Sci. 1124 (2008) 1-38.

[4] Corbetta S, \& Shulman G L, Control of goal-directed and stimulus-driven attention in the brain, Nature Reviews. 3(3) (2002) 201-215.

[5] Damoiseaux J S, Beckmann C F, Sanz Arigita E J, Barkhof F, Ph. Scheltens, Stam C J, Smith S M, \& Rombouts S A R B, Reduced resting-state brain activity in the default network in normal aging, Cereb. Cortex. 18 (2008) 1856-1864.

[6] Fossati P, Hevenor S J, Graham S J, Grady C, Keightley M L, Craik F \& Mayberg H, In search of emotional self: an fMRI study using positive and negative emotional words, Am J Psychiatry. 160 (2003) 1938-1945.

[7] Gao W, Zhu H, Giovanello K S, Smith J K, Shen D, Gilmore J H \& Lin W, Evidence on the emergence of the brain's default network from 2-week-old to 2-year-old healthy paediatric subjects, PNAS. 106(16) (2009) 6790-6795.

[8] Garrity A G, Pearlson G D, McKiernan K, Lloyd D, Kiehl K A \& Calhoun V D, Aberrant default mode functional connectivity in schizophrenia, Am J Psychiatry. 164 (2007) 450-457.

[9] Geday J \& Gjedde A, Attention, emotion, and deactivation of default activity in inferior medial prefrontal cortex, Brain and Cognition. 69 (2009) 344-352.

[10] Greicius M D, Krasnow B, Reiss A L, \& Menon V, The functional connectivity in the resting brain: a network analysis of the default mode hypothesis, PNAS. 100 (2003) 253-258. [11] Grimm S, Boesiger P, Beck Johannes, Schuepbach D, Bermpohl F, Walter M, Ernst J, Hell D, Boeker H, \& Northoff G, Altered negative BOLD responses in the default-mode 
Emotional faces and the default mode network Sreenivas, Boehm, \& Linden

network during emotional processing in depressed subjects, Neuropsychopharmacology. 34 (2009) 932-943.

[12] Gruberger M, Ben-Simon E, Levkovitz Y, Zangen A \& Hendler T , Towards a neuroscience of mind-wandering, Front. Hum. Neurosci. 5 (2011) 56.

[13] Gujar N, Yoo S, Hu, P \& Walker M P, The unrest resting brain: Sleep deprivation alters activity within the default-mode network, J Cognitive Neuroscience. 22 (2009) 1637-1648. [14] Gusnard D A, \& Raichle M E, Searching for a baseline: Functional imaging and the resting human brain, Nature Neuroscience. 2 (2001) 685-694.

[15] Gusnard D A, Akbudak E, Shulman G L \& Raichle M E, Medial prefrontal and self referential mental activity: relation to a default mode of brain function, PNAS. 98(7) (2001) 4259-4264.

[16] Kochan N A, Valenzuela M, Slavin M J, McCraw S, Sachdev P S \& Breakspear M, Impact of load-related neural processes on feature binding in visual working memory, PLoS ONE. 6(8) (2011) e23960.

[17] Laufs H, Krakow K, Sterzer P, Eger E, Beyerle A, Salek-Haddadi A, \& Kleinschmidt A, Electroencephalographic signatures of attention and cognitive default modes in spontaneous brain activity fluctuations at rest, PNAS. 100(19) (2003) 11053-11058.

[18] Linden D E J, Prvulovic D, Formisano E, Völlinger M, Zanella F E, Goebel R, \& Dierks T, The functional neuroanatomy of target detection: An fmri study of visual and auditory oddball tasks, Cereb. Cortex. 9 (8) (1999) 815-823.

[19] McKiernan K A, Kaufman J N, Kucera-Thompson J \& Binder J R, A parametric manipulation of factors affecting task-induced deactivation in functional neuroimaging, $J \operatorname{Cog}$ Neuroscience. 15(3) (2003) 394-408.

[20] Mannell M V, Franco A R, Calhoun V D, Canive J M, Thoma R J \& Mayer A R, Resting state and task-induced deactivation: A methodological comparison in patients with schizophrenia and healthy controls, Human Brain Mapping. 31 (2010) 424-437. 
Emotional faces and the default mode network Sreenivas, Boehm, \& Linden

[21] Mason M F, Norton M L, Van Horn J D, Wegner D M, Grafton S T \& Macrae C N, Wandering minds: the default network and stimulus independent thought, Science. 315(5810) (2007) 393-395.

[22] Mayer J S, Roebroeck A, Maurer K, \& Linden D E, Specialization in the default mode Task-induced brain deactivations dissociate between visual working memory and attention, Human Brain Mapping. 31 (2010) 126-139.

[23] Mayer J S, Bittner R A, Nikolic D, Bledowski C, Goebel R, \& Linden D E J, Common neural substrates for visual working memory and attention, Neuroimage. 36 (2007) 441-453. [24] Raichle M E, MacLeod A M, Snyder A Z, Powers W J, Gusnard D A \& Shulman G L, A default mode of brain function, Proceedings of the National Academy of Sciences, U.S.A. 98 (2001) 676-682.

[25] Reske M, Habel U, Kellermann T, Backes V, Shah N J, von Wilmsdroff M, Gaebel W, Zilles K, \& Schneider F, Differential brain activations during facial emotion discrimination in first-episode schizophrenia, Journal of Psychiatric Research. 43 (2009) 592-599. [26] Rotarska-Jagiela A, van de Van V, Oertel-Knochel V, Uhlhaas P J, Vogeley K, \& Linden D E J, Resting-state functional network correlates of psychotic symptoms in schizophrenia, Schizophrenia Research. 117 (2010) 21-30.

[27] Shim G, Oh J S, Jung W H, Jang J H, Choi C, Kim E, Park H, Choi J, Jung M H \& Kwon J S, Altered resting-state connectivity in subjects at ultra-high risk for psychosis: an fMRI study, BBF. 6 (2010) 58.

[28] Taylor S F \& Liberzon I, Neural correlates of emotion regulation in psychopathology, Trends Cogn Sci. 11 (2007) 413-418.

[29] Van Horn J D, The new perspectives in fMRI research award: exploring patterns of default-mode brain activity, Journal of cognitive neuroscience. 16(9) (2004) 1479-1480. [30] Wiebking C, de Greck M, Duncan N W, Heinzel A, Tempelmann C \& Northoff G, Are emotions associated with activity during rest or interoception? An exploratory fMRI study in healthy subjects, Neuroscience Letters. 491 (2011) 87-92. 
Emotional faces and the default mode network Sreenivas, Boehm, \& Linden

Figure Legends:

Figure 1: Upper panel: Brain areas with a main effect of stimulus category (deactivations): VMPFC, PC, Cuneus and LMFG; Lower panel: Brain areas with a main effect of stimulus category (Activations): RIFG, LIFG and LIPL. De/activations corrected for cluster threshold $=297 \mathrm{~mm}^{3}, \mathrm{p}<.01 . *$ represents the significant difference between de/activations for different emotions, $\mathrm{p}<.01,{ }^{* *} \mathrm{p}<.05$, Bonferroni corrected, (1) = Beta values extracted from whole-brain analysis, (2) = Beta values extracted from ROI analysis

Figure 2: Emotion specific neural connectivity based on the correlation analysis. The arrows indicate the identified relation between the ROIs. Each relation is specified with Pearson's $r$ and uncorrected $\mathrm{p}$ values. 
Emotional faces and the default mode network Sreenivas, Boehm, \& Linden

Tables

Table 1: Hit rate, mean RT from EFT behavioural response, mean and standard deviation on SAM ratings for arousal and valence from the supplementary behavioral data

\begin{tabular}{llllll}
\hline & Happy & Neutral & Sad & Angry & Fearful \\
\hline Hit rate & 71 & 81 & 72 & 77 & 68 \\
Mean RT (ms)** & 584 & 731 & 831 & 777 & 696 \\
Arousal M(SD) & $0.58(1.41)$ & $-2.51(1.38)$ & $-0.93(1.4)$ & $0.49(1.24)$ & $1(1.47)$ \\
Valence M(SD) & $2.54(0.66)$ & $-0.14(0.44)$ & $-1.69(0.71)$ & $-1.04(1.19)$ & $-1.09(1.27)$ \\
\hline
\end{tabular}

$* M(S D)=$ Mean $($ Standard deviation $) * * m s=$ millisecond .

Table 2: Areas showing a main effect of stimulus category.

\begin{tabular}{|c|c|c|c|c|c|}
\hline$\overline{\text { Region Of Interest }}$ & No. of Voxels* $\mathrm{X}$ & & $\overline{\mathrm{Y}}$ & $\bar{Z}$ & Significant Contrasts** \\
\hline VMPFC & 1913 & 1 & 56 & 9 & Happy-Neutral, Happy-Sad, Happy-Fear \\
\hline $\mathrm{PC}$ & 318 & 0 & -32 & 39 & Happy-Anger \\
\hline Cuneus & 3582 & -3 & -52 & 16 & Sad-Happy, Fear \\
\hline LMFG & 313 & -28 & 24 & 40 & Sad- Happy, Neutral, Anger, Fear \\
\hline RIFG & 1710 & 46 & 27 & 22 & Happy-Neutral, Sad, Fear \\
\hline LIFG & 492 & -50 & 17 & 23 & Happy-Neutral, Fear \\
\hline LIPL & 514 & -46 & -39 & 40 & Neutral-Happy, Fear \\
\hline
\end{tabular}



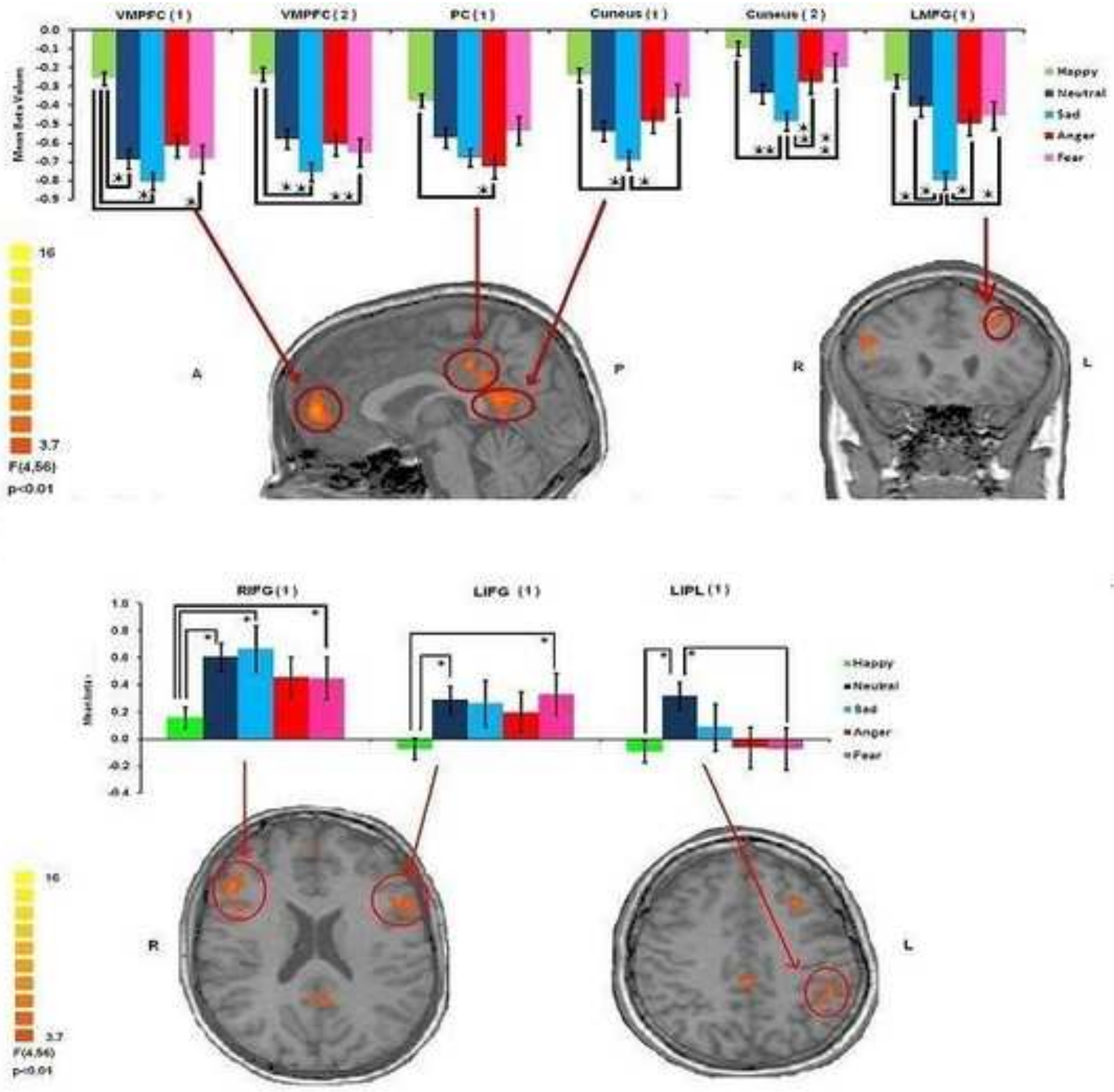


\section{HAPPY}

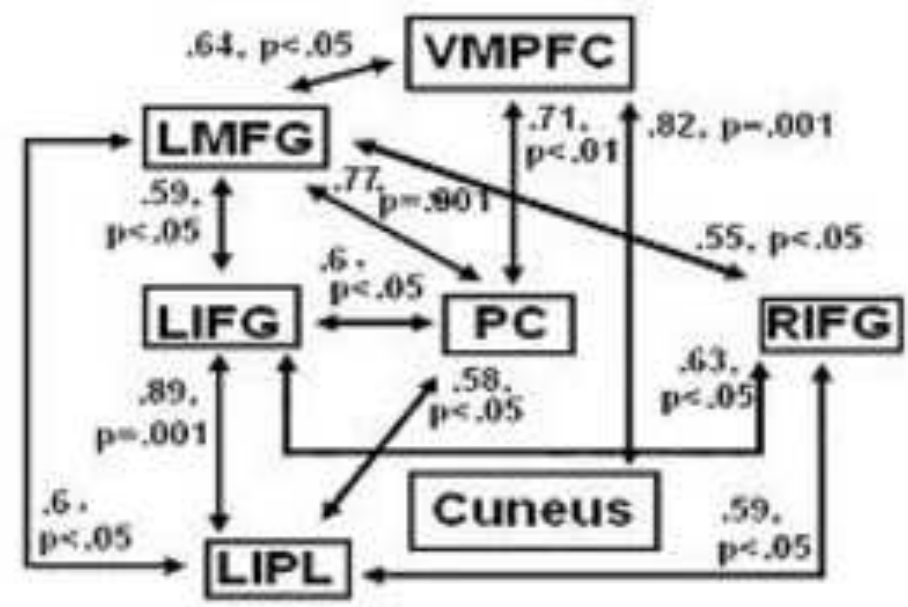

SAD

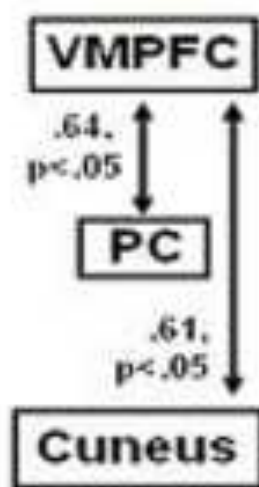

ANGRY

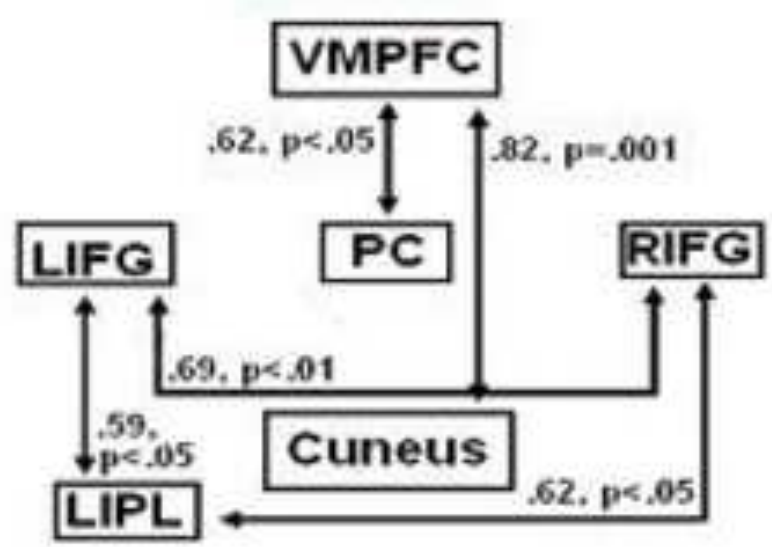

\section{NEUTRAL}

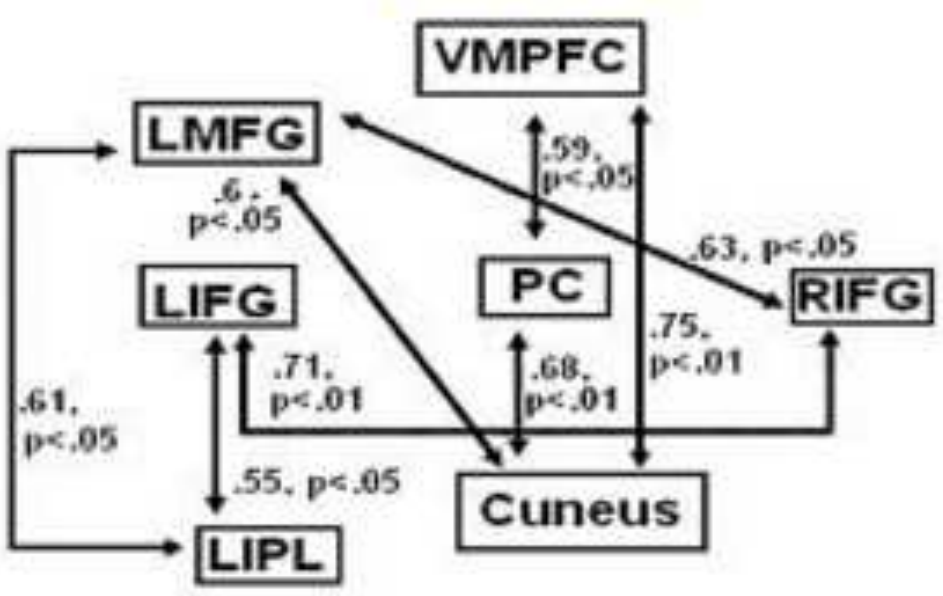

FEAR

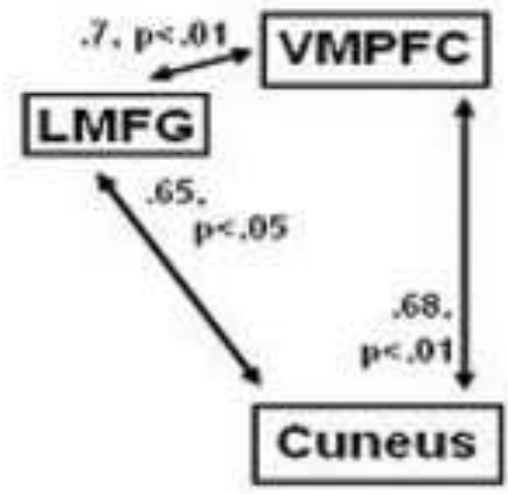

